\title{
The bigger the better? Konzeptionelle Konsequenzen der Erweiterung für die EU als internationaler Akteur
}

\author{
Johannes Varwick*
}

\begin{abstract}
Due to enlargement, the European Union has to change itself fundamentally. One single model of integration for all the 25 member states is even more difficult to develop than before. If this is true, the acting of the EU in international politics will be affected as well. On the one hand, the enlargement processes will strengthen the potential weight of the EU. On the other hand, the ability to act will be weakened because of the growing heterogeneity and a bigger spread of interests. The article analyses the consequences of the enlargement of the EU as an international actor and discusses the question which future models of integration are thinkable and likely.
\end{abstract}

Keywords: Enlargement, EU as an international actor, future of European integration

\section{Skizzen zur europapolitischen Problemagenda}

$\mathrm{D}$ ie politische Landkarte Europas hat sich in den vergangenen Jahren nachhaltig verändert, aber erst mit einem wohl unvermeidbaren Zeitverzug beginnen sich die neuen Strukturen mitsamt ihren vielschichtigen Konsequenzen auch auf der kognitiven Landkarte der Europäer einzuprägen. ${ }^{1}$ Insbesondere hat die Europäische Union (EU) als zentrale Organisation in Europa darüber zu entscheiden, ob sie sich von einem kraftvollen ökonomischen Akteur mit gemeinsamer Währung und einem umfassenden und täglich wachsenden gemeinschaftlichen Besitzstand (acquis communautaire) zu einem ebenso kraftvollen politischen und sicherheitspolitischen Akteur wandeln will und kann. Die EU steht damit vor der Entscheidung, ob sie sich hauptsächlich mit sich selbst beschäftigen will, oder aber ob sie bereit und in der Lage ist, friedenspolitische Stabilisierungsfunktionen für das internationale System auch über ihr eigenes Territorium hinaus zu übernehmen und mithin eine aktivere weltpolitische Rolle zu spielen.

All dies vollzieht sich vor dem Hintergrund der größten Erweiterung ihrer Geschichte. Die EU hat damit die Chance, ihre Erfolgsgeschichte in weiten Teilen Mittel- und Osteuropas fortzuschreiben und schrittweise den gesamten Kontinent zu stabilisieren und zu befrieden. Nachdem zum 1. Mai 2004 zunächst zehn Länder beigetreten sind, ist in den kommenden Jahren mit weiteren Beitritten zu rechnen Bulgarien, Rumänien und der Türkei wurde bereits eine konkrete Beitrittsperspektive zugesagt, und auch BosnienHerzegowina, Kroatien, Jugoslawien und Albanien werden sich nicht auf Dauer außen vor halten lassen. Es zeichnet sich also eine EU mit bis zu 30 Mitgliedstaaten ab. Diese historische Entscheidung ist alternativlos, hat aber gewichtige Konsequenzen. So werden die Interessenunterschiede bei steigender Mitgliederzahl zunehmen, die Diskussionen in den Gremien langatmiger und die Entscheidungsprozesse

\footnotetext{
* Prof. Dr. Johannes Varwick lehrt Politikwissenschaft mit dem Schwerpunkt Europäische Integration und internationale Organisationen an der punkt Europäische Integration und
Christian-Albrechts-Universität Kiel.

1 Als Problemaufriss siehe Johannes Varwick/Wilhelm Knelangen (Hrsg.): Neues Europa - alte EU? Fragen an den europäischen Integrationsprozess,
} Opladen 2004. komplexer. Ist politische Integration damit letztlich ein regionalistisches Konzept, das nur so lange praktikabel ist, wie ein gewisser Grenzwert eines sich vergrößernden Gebildes nicht überschritten wird? Funktioniert Integration nach dem EU-Modell nur so lange, wie es ein mehr oder weniger klar definiertes Außen und Innen gibt? Lässt sich in einer radikal erweiterten EU die friedensstiftende Funktion der europäischen Einigung aufrechterhalten oder bedeutet dies langfristig den Zerfall in eine gehobene Freihandelszone? Wie steht es angesichts dieser Fragen um die außen- und sicherheitspolitische Handlungsfähigkeit der erweiterten EU? ${ }^{2}$

Die EU wird sich im Zuge dieses Prozesses fundamental ändern bzw. ändern müssen, und ein einheitliches Integrationsmodell für alle 30 Mitgliedstaaten wird immer schwieriger zu finden sein. Dieser Befund wird auch das Auftreten der EU auf der internationalen Bühne verändern. Ebenso wie die Erweiterung das potentielle Gewicht der EU stärkt, schwächt die Erweiterung gleichzeitig die Handlungsfähigkeit der EU durch eine zunehmende Heterogenisierung. ${ }^{3}$ In diesem Beitrag werden die Konsequenzen der Erweiterung für die außen- und sicherheitspolitische Handlungsfähigkeit der Union analysiert und mit der Frage verbunden, welche zukünftigen Integrationsmodelle denkbar und wahrscheinlich sind.

\section{Implikationen der Erweiterung für die GASP/ESVP}

Im außen- und sicherheitspolitischen Bereich sind zwar in den vergangenen Jahren enorme Fortschritte erzielt worden und im Vergleich zum Integrationsstand von Anfang der 1990er Jahre ist der Status quo trotz aller Differenzen bemerkenswert. Gleichwohl reichen die Regelungen in den Verträgen und die teilweise blumigen Absichtserklärungen in den Dokumenten der EU oftmals weiter als der am prakti-

2 Ausführliche Argumentation bei Johannes Varwick: EU-Erweiterung: Stabilitätsexport oder Instabilitätsimport?, in: Aus Politik und Zeitgeschichte (1-2) 2002, S. 23-30

3 Vgl. Helga Haftendorn: Sicherheitspolitik im strategischen Dreieck »Berlin - Paris - Washington«, in: Politische Vierteljahresschrift (1) 2004, S. 1-8, hier S. 7. 
schen output zu messende politische Wille zur Kooperation. Hierfür lassen sich zahlreiche Beispiele anführen, von wichtigen strategischen Aspekten wie der Uneinigkeit über einen europäischen Sitz im Sicherheitsrat der Vereinten Nationen oder der Frage, in welchem Verhältnis eine militärische europäische Eingreiftruppe zur NATO stehen soll, bis hin zu jeweils aktuellen außenpolitischen Streitfragen. So hat insbesondere der Irak-Krieg 2002/2003 abermals die unterschiedlichen außen- und sicherheitspolitischen Vorstellungen der EU-Staaten gezeigt. Der Riss verlief gleichwohl nicht nur zwischen alten und neuen (bzw. zukünftigen) Mitgliedstaaten, sondern ging mitten durch die EU. ${ }^{4}$

Doch damit nicht genug. Es lassen sich auch grundsätzlichere Fragen an die EU als internationaler Akteur formulieren. Will die EU internationale Politik strukturprägend gestalten oder will die EU gestaltet werden? Welches Rollenkonzept liegt der Gemeinsamen Außen- und Sicherheitspolitik zu Grunde? ${ }^{5}$ Was will die EU mit welchen Mitteln erreichen? Wird es in Zukunft noch möglich sein, ein »Gemeinsames Drittes « zu formulieren, das jenseits aller Kontroversen im Einzelnen die Möglichkeit zur Verständigung bietet? Was ist die gemeinsame Grundlage, von der aus europäische Politik formuliert wird? Bleibt Europa nach wie vor Antwort auf schreckliche Erfahrungen, oder wird es zunehmend zu einer »Selbstbedienungs-Agentur «, bei der die Mitglieder lediglich die Vorteile einheimsen, aber bei ernsten Krisen nicht bereit sind, kurzfristige nationale Interessen zu Gunsten längerfristiger Gemeinschaftsinteressen (die dann in aller Regel allen zu Gute kommen) zurückzustellen? Noch deutlicher formuliert: Setzt sich stärker als bisher eine »Sparkassenmentalität« durch, bei der die EU als eine Art Kasse gesehen wird, aus der möglichst viel kurzfristige Rendite zu ziehen ist (Stichworte: Nettozahlerdebatte oder Agrarbeihilfen), die politisch-strategischen Vorteile einer stabilen Friedensgemeinschaft dabei aber nicht mehr ins Gewicht fallen?

\subsection{Vertiefung vor Erweiterung?}

Eine Vertiefung der EU - das heißt die Entwicklung einer tragfähigen konstitutionellen Grundlage und die Reform der Entscheidungsprozesse - galt in Politik und Wissenschaft als Voraussetzung für die Erweiterung. Ein Weitermachen nach dem bisherigen Integrationsmodell ist erklärtermaßen an die Grenzen des Machbaren gestoßen. Auf der Agenda der zu diesem Zweck begonnenen Verfassungsberatungen des dafür eingesetzten Europäischen Konvents stand u.a. eine bessere Verteilung und Abgrenzung der Zuständigkeiten, eine Neuordnung der bisherigen Verträge, eine Demokratisierung und Effizienzsteigerung der EU sowie die Frage nach den Bestandteilen einer künftigen europäischen Verfassung. Im Kern ging und geht es dabei um die Frage, welcher Ebene künftig die zentralen Kompetenzen für die Gestaltung des politischen, wirtschaftlichen und gesellschaftlichen Lebens

4 Es sei nur daran erinnert, dass z.B. Frankreich, Deutschland und Belgien vehement gegen das militärische Eingreifen im Irak waren, während etwa vehement gegen das militärische Eingreifen im Irak waren,
Großbritannien, Spanien und Italien die USA unterstützten.

$5 \mathrm{Zu}$ dieser zentralen Frage, die in diesem Beitrag allerdings nicht behandelt werden kann, siehe: Hans-Georg Ehrhart: What model for CFSP, Paris 2002 (Chaillot Paper 55). in Europa zukommen sollen. Nach dem vorläufigen Scheitern der Bemühungen um einen europäischen Verfassungsvertrag im Dezember 2003 ist die weitere Entwicklung derzeit noch nicht absehbar. Nach dem spanischen Regierungswechsel im Frühjahr 2004 ist zwar Bewegung in die Diskussion gekommen und die Staats- und Regierungschefs wollen sich noch unter irischer Präsidentschaft bis Sommer 2004 auf einen gemeinsamen Vertragstext einigen. Doch selbst wenn das Paket von den zur Entscheidung befugten Staats- und Regierungschefs als »Herren der Verträge « nicht wieder aufgeschnürt würde und alle dann 25 Mitgliedstaaten den neuen Text ratifiziert haben, bleibt es umstritten, ob damit der große Wurf für die zukünftige Gestalt der EU gefunden wäre.

Diese grundsätzliche Debatte lässt die GASP/ESVP nicht unberührt. So enthielt der Verfassungsentwurf - entgegen der ursprünglichen Planung - Vorschläge in den Bereichen der GASP und ESVP. ${ }^{6}$ Dazu zählen der Posten eines Europäischen Außenministers (Artikel 27) und die Aufnahme einer kollektiven Verteidigungsklausel (Artikel 40, Absatz 7), die die Möglichkeit der Errichtung eines verteidigungspolitischen Kerns in der EU schaffen würde. Bemerkenswert ist auch die vorgeschlagene Einführung einer strukturierten sicherheits- und verteidigungspolitischen Zusammenarbeit einer Gruppe von Mitgliedstaaten (Artikel 40, Absatz 6). Auf diesem Wege soll das Instrument der verstärkten Zusammenarbeit auch auf die ESVP ausgeweitet werden, ein Schritt, der noch in Nizza am britischen Widerstand gescheitert war (Artikel III-213, Absatz 5). Auf absehbare Zeit wird aber der Vertrag von Nizza die Geschäftsgrundlage für die EU sein. Eine Vertiefung (d.h. die Entwicklung einer tragfähigen konstitutionellen Grundlage und die Reform der Entscheidungsprozesse) galt geradezu als Voraussetzung für die Erweiterung. Nun wird man zunächst ohne eine Verfassung (aber mit der Rechtsgrundlage des Vertrags von Nizza) und ohne die notwendigen Reformen die Erweiterung vollziehen und damit ein Experiment mit ungewissem Ausgang eingehen müssen. Was dies für die Bestandsfähigkeit der EU bedeutet, ob damit ein schleichender Zerfall eingeleitet worden ist oder ob sich neue Integrationsformen jenseits der bestehenden Verträge entwickeln werden, muss ebenso abgewartet werden wie die Frage, was dies für die Möglichkeiten einer europäischen Außen- und Sicherheitspolitik bedeutet.

\subsection{Veränderte Akteursqualität der erweiterten EU?}

Die Erweiterung wird die jungen Demokratien festigen und 450 Millionen Menschen werden einen gemeinsamen Binnenmarkt sowie einen gemeinsamen Raum der Freiheit, des Rechts und der Sicherheit bilden. Die Bevölkerung der EU wird um rund 20 Prozent zunehmen, die Wirtschaftsleistung aber zunächst nur um rund fünf Prozent steigen. So vergrößert sich die Bevölkerung der EU zum Mai 2004 von rd.

\footnotetext{
6 Dazu ausführlich Martin Koopmann: Notwendige Fortschritte, verpasste Chancen: die GASP/ESVP im Verfassungsentwurf des Europäischen Konvents, in: Hans-Georg Ehrhart/Burkard Schmitt (Hrsg.): Die Sicherheitspolitik der EU im Werden. Bedrohungen, Aktivitäten, Fähigkeiten, Baden-Baden 2004, S. 78-90.
} 
374,4 Mio. auf 449 Mio., das Bruttoinlandsprodukt nimmt gleichwohl zunächst nur um 437 Mrd. Euro (das entspricht etwa der Wirtschaftskraft der Niederlande) auf $9598 \mathrm{Mrd}$. zu. Allerdings weisen die neuen Mitglieder durchweg höhere Wachstumsraten als die Altmitglieder auf, sollte dieser Trend andauern, wird das ökonomische Potential der gesamten EU steigen und der Abstand der Neumitglieder geringer werden. Die direkten zusätzlichen ökonomischen Wachstumseffekte durch die Erweiterung werden für die gesamte EU allerdings zunächst auf lediglich 0,005 bis 0,2 Prozent pro Jahr geschätzt. $^{7}$

Analysen der Haltung der mittelosteuropäischen Neu-EUStaaten zu den Inhalten der GASP lassen im längerfristigen Trend eine erhebliche Angleichung erkennen. So stieg die Übereinstimmung mit den allgemeinen Stellungnahmen im Rahmen der GASP von 25,5 Prozent im Jahr 1995 auf 71,8 Prozent im Jahr 2002. Allerdings ergibt die Zustimmung zu dem formalisierteren Instrument der »Gemeinsamen Standpunkte« im Rahmen der GASP ein differenziertes Bild. Hier schwankt die Zustimmungsrate erheblich und betrug im Jahr 2002 lediglich 39,2 Prozent, während sie 2001 (55 Prozent) und 2000 (52,9 Prozent) schon höher lag. ${ }^{8}$ Eine Vergrößerung des spread of interests in der EU ist aber dennoch wahrscheinlich. Dies gilt selbst angesichts der plausiblen Erwartung, dass die neuen Mitglieder gewissermaßen in den außenpolitischen common sense der EU - sofern es ihn denn gibt - hineinsozialisiert werden und heftige Positionsunterschiede wohl nur in Einzelfällen zu erwarten sein werden.

Jenseits dieser konzeptionellen Fragen wird die Erweiterung aber auch inhaltlich die zukünftige Akteursqualität der EU verändern. Dies sei an drei Beispielen verdeutlicht:

- Die Mehrzahl der neuen Staaten sind kleine Staaten oder Kleinststaaten. So kommt Malta auf 300.000 und Estland auf 1,4 Millionen Einwohner. Mit Polen (38,6 Millionen) tritt lediglich ein Staat bei, der hinsichtlich der Bevölkerungsgröße etwa auf das Gewicht Spaniens kommt. Im Ergebnis werden die großen Staaten noch stärker als bisher versucht sein, mit Absprachen unter einander Führungsfähigkeit im Sinne einer Art Direktorat wahrzunehmen. Ebenso sicher werden die kleinen Staaten darauf jedoch mit Unmut reagieren und möglicherweise Koalitionen auch außerhalb der EU suchen und finden.

- Die EU 25 wird einen gemeinsamen Grenzverlauf mit Russland, Weißrussland, der Ukraine, Mazedonien, Albanien, Serbien und Montenegro sowie Kroatien haben. Wenn im Jahr 2007 Bulgarien und Rumänien EUMitglieder sein werden, käme eine Grenze mit Moldawien dazu. Im Falle einer Mitgliedschaft der Türkei würde die EU zusätzlich an Georgien, Armenien, Iran, Irak und Syrien grenzen. Bereits nach der Erweiterungsrunde im Mai 2004 wird die GASP sich stärker als bisher mit einer

7 So die Schätzung des Hamburger Weltwirtschaftsarchivs, vgl. FAZ vom 18.4.2004, S. 18.

8 Zahlen nach Mathias Jopp/Barbara Lippert/Elfriede Regelsberger: Europäische Außen- und Sicherheitspolitik der erweiterten Union - interne und externe Herausforderungen an Politik und Institutionen in GASP und ESVP, in: Matthias Chardon u.a. (Hrsg.): Regieren unter neuen Herausforderungen: Deutschland und Europa im 21. Jahrhundert, Baden-Baden 2003, S. 253-265, hier S. 258. neuen Nachbarschaftspolitik nach Osteuropa beschäftigen müssen. ${ }^{9}$ Die Neumitglieder bringen einen anderen Blickwinkel sowie andere Netzwerke und Perspektiven in die EU ein und dies wird sicherlich Konsequenzen für die außen- und sicherheitspolitische Agenda haben. Auch das Verhältnis zu Russland wird vor neuen Herausforderungen stehen - so ist Kaliningrad nunmehr eine russische Exklave inmitten der EU und die möglichen ökonomischen Nachteile Russlands durch die EUMitgliedschaft wichtiger Handelspartner verlangen ein intensiveres Eingehen auf russische Interessen. Probleme ergeben sich auch an den zukünftigen »SchengenGrenzen«, die zwar seitens der EU nicht als undurchlässige Blockgrenze definiert werden, aber dennoch von den nicht der EU angehörenden Staaten als solche gesehen und erlebt werden könnten.

- Insbesondere aber stützt die Erweiterung eine transatlantische Orientierung der EU. Dies zeigte sich unter anderem an der Haltung zum Irak-Krieg 2002/2003, bei der alle acht mittelosteuropäischen Beitrittskandidaten fest an der Seite der USA standen. Bei den wichtigen Akteuren der transatlantischen Sicherheitsbeziehungen bestehen aber ganz offensichtlich auch unterschiedliche Vorstellungen hinsichtlich des zukünftigen Verhältnisses von NATO und EU. Während Großbritannien traditionell eine enge Anlehnung an die USA - für die die NATO allein als vetoberechtigter Pfeiler einer Zwei-Pfeiler-Allianz akzeptabel scheint - bevorzugt und mit einem »bandwagoning « versucht, Einfluss auszuüben, ist es traditionelle französische Politik, eher im Sinne eines »balancing « eine Gegenmacht zu den USA aufzubauen. Polen tendiert eindeutig und unmissverständlich zur britischen Position und wird darin von allen neuen EU-Staaten unterstützt. ${ }^{10}$ Nach der Erweiterung der NATO auf 26 Mitgliedstaaten ist eine weitgehende Mitgliederkongruenz zwischen EU und NATO gegeben. Lediglich sechs Staaten (Finnland, Irland, Malta, Österreich, Schweden und Zypern) sind Mitglied in der EU, aber nicht in der NATO, während fünf Staaten (Island, Norwegen, Kanada, die USA und die Türkei) NATO-Mitglieder sind, die nicht der EU angehören.

\section{Flexibilisierung und Finalitätsdebatte: Konsequenzen für die EU-Außenpolitik}

Eine mögliche Antwort auf die zunehmende Heterogenität und die Herausforderung der Handlungsfähigkeit einer erweiterten EU ist das Prinzip der »Verstärkten Zusammenarbeit «, das in Wissenschaft und Politik unter ganz verschiedenen Bezeichnungen (u.a. flexible, abgestufte, differenzierte Integration) als ein wichtiges Strukturprinzip der Zukunft diskutiert wird. Damit steht ein Integrationsszenario auf der politischen Agenda, das die EU nachhaltig verändern wird. Bereits in der so genannten Flexibilitätsklausel des Nizza-

\footnotetext{
9 Siehe dazu: Martin Kahl: Welche Grenzen für Europa?, in: Varwick/ Knelangen (Anm. 1), S. 133-148

10 Siehe dazu Johannes Varwick: Die Zukunft der transatlantischen Sicherheitsbeziehungen. Vom Partner zum Rivalen?, in: Österreichische Militärische Zeitschrift (2) 2004, S. 141-148.
} 
Vertrags wurde festgelegt, denjenigen Mitgliedstaaten, die mit der Integration in bestimmten Politikfeldern weiter voranschreiten wollen als andere, dies unter Inanspruchnahme der gemeinschaftlichen Organe, Verfahren und Mechanismen zu gestatten. Es wird mithin stärker über eine zeitliche, sektorale, funktionale oder geografische Flexibilisierung der Integration nachgedacht. Damit verabschieden sich denkbare und praktikable Ordnungsmodelle aber von der Vorstellung einer einheitlichen und gleichzeitigen Integration aller betroffenen Nationalstaaten.

Unterschiedlichste Modelle dieser Differenzierung werden derzeit diskutiert, von der Aufbauflexibilisierung (die nur für neue Projekte - wie z.B. eine Verteidigungsunion - gelten soll) bis zur Bestandsflexibilisierung (die sich auf bereits vergemeinschaftete Politikfelder - wie etwa Teilbereiche der GASP - bezieht). Im Grundsatz sind drei flexible Integrationsformen zu unterscheiden, die vor allem hinsichtlich der Punkte Kriterium, Entscheidung, Motiv und Methode differieren. Jedes der drei Modelle birgt Risiken in sich. Während bei dem Modell der Abgestuften Integration die Gefahr einer dauerhaften Spaltung der Union zwar gegeben ist, aber versucht wird, durch ein gemeinsames Ziel einen gemeinsamen Rahmen zu erhalten, wird bei den Modellen der Variablen Geometrie und der À la Carte-Konzeption dieses gemeinsame Integrationsziel aufgegeben und stärker nach dem spezifischen Integrationswillen entschieden. Die Risiken liegen auch da auf der Hand. Bei dem Modell der Variablen Geometrie ist der Zusammenhalt der Union gefährdet und zudem drohen überkomplexe Entscheidungsstrukturen. Bei einem Europa à la Carte droht ein Zerfall in verschiedene Gemeinschaften, die dann kein Zusammengehörigkeitsgefühl mehr entwickeln können. Nachdem auf Grund der Erfahrungen mit dem Amsterdamer Vertrag Einigkeit bestand, dass die bisherigen vertraglichen Regelungen ungeeignet waren, hat der Vertrag von Nizza neue rechtliche Rahmenbedingungen geschaffen, von denen aber noch nicht zu prognostizieren ist, ob sie tatsächlich leichter zu handhaben sind. Die Regelungen - die auch im Verfassungsvertragsentwurf in diesem Sinne enthalten sind - orientieren sich tendenziell am Modell der Abgestuften Integration. Für die Zulässigkeit einer verstärkten Zusammenarbeit sind gemäß Art 40 - 44a EUV vier Rahmenbedingungen vorgesehen: Sie darf nur als letztes Mittel angewendet werden, muss prinzipiell allen Mitgliedern offen stehen, in den institutionellen EURahmen eingebunden sein und schließlich eine Mindestteilnehmerzahl von acht Staaten umfassen. Die Auslösung der verstärkten Zusammenarbeit kann im Rat mit qualifizierter Mehrheit gefasst werden. Damit sollen die Risiken der verstärkten Zusammenarbeit abgemildert, zugleich aber die Handlungsfähigkeit der Europäischen Union beibehalten werden. ${ }^{11}$

\footnotetext{
11 Ausführliche Argumentation bei: Johannes Varwick: Flexibilisierung oder
} Zerfall. Hat die EU Bestand?, in: Varwick/Knelangen (Anm. 1), S. 59-77.
Ob sich der Bereich Außen- und Sicherheitspolitik für eine Flexibilisierung eignet, wird kontrovers diskutiert. ${ }^{12}$ Während einerseits zu Recht darauf hingewiesen wird, dass dies der wohl einzige Weg sein wird, eine erweiterte EU im sicherheitspolitischen Bereich handlungsfähig zu halten, wird andererseits mit nicht weniger Recht auf die Risiken einer solchen Entwicklung hingewiesen. Auch der deutsche Außenminister Fischer fordert seit einiger Zeit - im Übrigen in Abkehr von seinen bisherigen europapolitischen Vorstellungen - die »strategische Dimension der europäischen Einigung « mehr in den Vordergrund zu rücken. Diese müsse angesichts der neuen weltpolitischen Realitäten Vorrang vor einer »kerneuropäischen Perspektive« haben, bei der eine kleine Gruppe von integrationsbereiten Staaten mit einer Vertiefung voranschreitet. ${ }^{13}$ Man hat den Eindruck, Fischer hat sich damit abgefunden, dass eine radikal erweiterte Union Abschied von einer föderalen Entwicklung (d.h. einer ebenso radikalen Vertiefung) nehmen muss. Vielmehr gehe es heute um die geostrategische Stabilisierung Europas und seiner weiteren Peripherie sowie um den Umgang mit neuen sicherheitspolitischen Herausforderungen, die sich nicht mit kleineuropäischen Lösungsansätzen bestehen ließen.

\subsection{Die Frage nach dem Endzustand der Integra- tion: Prognosen und Szenarien}

Es gehörte zu den Erfolgsgeheimnissen der europäischen Integration, dass die Mitgliedstaaten seit der Initialzündung der Montanunion im Jahr 1951 der Grundsatzfrage nach der Finalität des europäischen Integrationsprozesses auswichen. Denn mit der Entscheidung für die Methode der sektoralen Teilintegration, die bei der Schaffung der Europäischen Gemeinschaft für Kohle und Stahl Pate stand, erklärten sie sich zwar zur Übertragung nationaler Kompetenzen in einem zunächst eng umgrenzten Bereich bereit, ließen aber die Frage der Zielperspektive der Integration bewusst offen. Auch in der Folge konzentrierten sich die Verträge stattdessen darauf, im Einzelnen anzugeben, für welche Bereiche die europäische Ebene eine Befugnis besitzt und nach welchen institutionellen Regeln Entscheidungen getroffen werden. Wenn dieser Frage nun nach Auffassung der Staats- und Regierungschefs nicht mehr ausgewichen werden kann und soll, dann bedeutet dies tatsächlich die Chance, EU-Europa zu vollenden. Damit wird aber auch ein enormes Konfliktpotenzial sichtbar, das neben der grundsätzlichen Frage nach der maximal verkraftbaren Zahl an Mitgliedstaaten und unterschiedlichen Interessen auch daraus resultiert, dass sich die Mitglieder über die grundsätzliche Richtung der Integration nicht einig sind. Hinzu kommt ein sich verschärfender Konflikt zwischen großen und kleinen Mitgliedern.

\footnotetext{
12 Vgl. als Befürworter einer Avantgarde der Integrationswilligen in der GASP: Hans-Georg Ehrhart: Abschied vom Leitbild "Zivilmacht ". Konzepte zur EU-Sicherheitspolitik nach dem Irak-Krieg, in: Varwick/Knelangen (Anm, 1), S. 149-163, und als Skeptiker: Peter Schmidt: Wick/Knelangen (Ans 1), S. 149-163, und als Skeptiker. Peter Schmidt: Kerneuropa in der Sicherheitspolitik. Integration oder Spaltung der EU?,
in: Erich Reiter (Hrsg.): Jahrbuch für internationale Sicherheitspolitik 2003, Hamburg 2003, S. 241-256.

13 So Fischer etwa im Interview mit der Frankfurter Allgemeinen Zeitung
} vom 6.3.2004, S. 9. 
Zwar können Prognosen und Szenarien für verschiedene Entwicklungen skizziert werden, letztlich sind jedoch sowohl die externen politischen Herausforderungen als auch die internen Entwicklungen der EU nur begrenzt vorhersehbar. So ist an dieser Stelle auch keine Vorhersage über die Zukunft beabsichtigt. Vielmehr sollen die Szenarien aufzeigen, welche Entwicklungen künftig möglich sein könnten, unter welchen Voraussetzungen sie eintreffen und welche Implikationen sich daraus ergeben. Mit dieser wichtigen Einschränkung vorangestellt, lassen sich in idealtypischer Weise drei Szenarien für die künftige Entwicklung der Europäischen Union entwerfen: ${ }^{14}$

- Das Staatswerdungsszenario erwartet und fordert den "großen Sprung « nach vorne, ist allerdings äußert voraussetzungsreich. Denn ohne die Bereitschaft zur weiteren Abgabe staatlicher Souveränität ist eine solche Entwicklung nicht denkbar. Wenn die EU zum größten Erweiterungsschritt ihrer Geschichte antritt, dann wird es in dieser Perspektive entscheidend darauf ankommen, dass die beiden Säulen Erweiterung und Vertiefung ihre Balance behalten. Allein als gehobener Binnenmarkt würde eine erweiterte EU kaum funktionieren. So müsste von der Erweiterung früher oder später ein starker Druck in Richtung auf weitere Vertiefung ausgehen. Die EU entwickelt sich in dieser Perspektive mittelfristig zu einer »supranationalen Föderation $\aleph^{15}$ mit einer Verfassung, starken Gemeinschaftsorganen, klarem Kompetenzkatalog sowie demokratischen Kontroll- und Legitimationsverfahren. Die Problemlösungsfähigkeit einer solchen Union wäre wenn sie denn zu Stande käme - vergleichsweise hoch und Europa hätte als Zone des Friedens und der Stabilität auch erhebliche Gestaltungskraft nach außen. Die Frage nach der künftigen Gestalt der EU ist damit aber noch nicht beantwortet. Denn bei diesem Szenario bleiben zunächst mehr Fragen als Antworten. Ist der Grad an gemeinsamer politisch-kultureller Identität gegeben, der Voraussetzung für eine solche Entwicklung ist? Welche Rolle spielen die Mitgliedstaaten und welche Kompetenzen werden sie behalten? Begibt sich die EU eher auf den präsidentiellen oder auf den parlamentarischen Entwicklungspfad? Mit wie vielen Staaten lässt sich eine solche Entwicklung praktikabel gestalten? Was passiert mit den Staaten, die nicht bereit sind, bei diesem Konzept mitzumachen?

- Das Erosionsszenario nimmt hingegen an, dass die EU unter der Last der Erweiterung und den wachsenden Interessenunterschieden ihrer Mitgliedstaaten zusammenbricht oder schleichend erodiert. Die mit dem Ende des Ost-West-Konflikts veränderte politische Landschaft schlägt nunmehr - mehr als ein Jahrzehnt nach diesen tektonischen weltpolitischen Verschiebungen - voll auf den europäischen Integrationsprozess durch. So gibt es in dieser Perspektive Anzeichen dafür, dass jene Rivalitäten innerhalb Europas wieder auftauchen, die für ausgeräumt gehalten worden waren. Die Gemeinschaftsinstitutionen

14 Siehe dazu Franco Algieri/Janis A. Emmanouilidis/Roman Maruhn: Europas Zukunft. Fünf EU-Szenarien, München 2003.

15 Armin von Bogdandy: Die Europäische Union als supranationale Föderation, in: Integration (2) 1999, S. 95-112. sind in dieser Perspektive zu schwach, um als ausgleichendes Element zu wirken. Unter der Prämisse, dass die nationalstaatliche Ebene in einer wachsenden Zahl von Politikfeldern für die Lösung der zahlreichen Probleme nicht mehr der geeignete Ort ist, hat dieses Szenario für die Problemlösungsfähigkeit der Politik äußerst negative Auswirkungen. Denn wenn die europäische Ebene erodiert und kein angemessener Ersatz zur Verfügung steht, werden die Nationalstaaten auf Alleingänge setzen und früher oder später in einen konfliktträchtigen Wettbewerb untereinander geraten. Europa würde zum Raum der Instabilität werden und dementsprechend auch keinen Beitrag zur Lösung der außereuropäischen Sicherheitsprobleme leisten können.

- Das Muddling-Through-Szenario geht davon aus, dass die Europäische Union im Großen und Ganzen das bleibt, was sie nach dem Integrationsstand zwischen NizzaVertrag und Verfassungskonvent ist: ein unvollkommenes, reformbedürftiges, kompliziertes politisches Gebilde sui generis, das dennoch für seine Mitgliedstaaten und seine Bürgerinnen und Bürger wie auch für die Stabilität des Kontinents unverzichtbar ist. Die europäische Ebene hat staatsähnliche Kompetenzen und gleichzeitig wird ihr von den Mitgliedstaaten die Staatsqualität abgesprochen. Die Kraft zu einem den Erfordernissen angemessenen Reformschritt bringen die Mitgliedstaaten in dieser Perspektive gleichwohl nicht auf, es wird aber im Grundsatz allseits akzeptiert, dass ohne die EU in nahezu allen Politikfeldern kein effektives und effizientes Regieren mehr möglich ist. Innerhalb dieses Szenarios bleibt offen, ob das integrationspolitische Pendel zu mehr gemeinschaftlichen Lösungen oder zu einer Abkehr von der Integration ausschlagen wird. So wäre eine gewisse Rückverlagerung von Kompetenzen auf die Mitgliedstaaten ebenso denkbar wie die engere Zusammenarbeit einzelner Mitgliedstaaten diesseits oder jenseits des EU-Vertrags. Das Auftreten der EU auf internationaler Bühne bleibt so wie bisher: unvollkommen, widersprüchlich, aber letztlich doch für die Mitgliedstaaten unverzichtbar.

Von allen drei Szenarien ist die Eintreffwahrscheinlichkeit des Muddling-Through-Szenarios als am höchsten einzuschätzen. In welcher Variante es sich durchsetzen wird, dürfte sehr stark von der Haltung wichtiger Mitgliedstaaten wie auch von den externen Faktoren - die sich aus den Unwägbarkeiten der internationalen Politik ergeben - abhängen.

\subsection{Bilanz: Die Grenzen der Integration}

Was folgt aus dieser Einschätzung für die Ausgangsüberlegung dieses Beitrags nach den Konsequenzen für die Bemühungen um eine effektive GASP/ESVP? Angesichts des erreichten Integrationsstandes in anderen Politikbereichen, der Schicksalsgemeinschaft einer gemeinsamen Währung und der anspruchsvollen Problembereiche in der internationalen Politik würde ein Scheitern der Bemühungen um europäische Außen- und Sicherheitspolitik das gesamte Projekt 
Europäische Union gefährden. ${ }^{16}$ Die gemeinsame außenpolitische Stimme Europas muss also nicht nur gesucht und in Sonntagsreden beschworen, sondern endlich auch gefunden werden. Was notwendig wäre, ist jedoch noch nicht mehrheitsfähig: die radikale Verlagerung sicherheitspolitischer Souveränität und Loyalität weg von den Hauptstädten bzw. die Einführung von Mehrheitsentscheidungen auch im Bereich der GASP/ESVP. Dennoch bleibt der ambivalente Charakter der GASP/ESVP zwischen Integration und nationaler Souveränitätswahrung erhalten. Der Weg ist also weiterhin steinig, aber er dürfte inzwischen unumkehrbar sein. EUEuropa wird mehr für seine eigene Sicherheit verantwortlich sein als jemals zuvor und die europäische Politik muss die Voraussetzungen dafür verbessern, diese Rolle auch auszufüllen. Die Europäische Union wird ein »Staatenverein in einer Welt von Staaten bleiben. Das Zeug zur Utopie hat sie nicht, aber es ist noch nicht lange her, da hätte sie niemand zu träumen gewagt. ${ }^{17}$ Doch heute geht es weniger um die

$16 \mathrm{Zu}$ diesem Zusammenhang siehe Johannes Varwick: Die ESVP: eine folgerichtige Weiterentwicklung der GASP?, in: Werner Hoyer/Gerd Kaldrack (Hrsg.): Europäische Sicherheits- und Verteidigungspolitik. De Weg zu integrierten europäischen Streitkräften?, Baden-Baden 2002, S. 96-107.

17 Joscha Schmierer: Mein Name sei Europa. Einigung ohne Mythos und Utopie, Frankfurt/M. 1996, S.207.
Verwirklichung der Utopie eines befriedeten und friedlichen Kontinents Europa (obgleich dieser Gedanke nichts an Aktualität verloren hat), sondern auch um die Gestaltungskraft der EU in der Welt des 21. Jahrhunderts. Und um diese Gestaltungskraft sieht es aller Wahrscheinlichkeit unter den gegebenen Vorraussetzungen europäischer Politik eher bescheiden aus.

Denn ein schleichender Zerfall der EU ist ebenso wenig auszuschließen wie die Entwicklung einer völlig neuen Integrationsform jenseits der bestehenden Verträge. Obgleich sich der Bereich Außen- und Sicherheitspolitik wie kaum ein anderes Politikfeld für gemeinschaftliche Lösungen geradezu aufdrängen würde, steht nicht zu erwarten, dass mit 25 oder gar 30 Mitgliedstaten das gelingen könnte, was schon mit 15 Staaten nicht erreicht werden konnte: die wirksame Einbringung einer gemeinsamen europäischen Stimme in die internationale Politik. Dabei wird den Europäern, die im Übrigen von außen schon sehr viel mehr als ein gemeinsamer Akteur wahrgenommen und nachgefragt werden, als dies von innen erkennbar wäre, schon lange nicht mehr gestattet, sich auf interne Nabelschau und innere Streitereien zu beschränken. Die schwierigen Debatten über die Zukunft der Integration wie auch der europäischen Außen- und Sicherheitspolitik stehen der EU erst noch bevor.

\title{
Europäische Sicherheitspolitik am Scheideweg
}

\author{
Hans J. Giessmann*
}

\begin{abstract}
The accession of seven East and Central European states to NATO, and the entry of ten states to the EU, represent an unprecedented peaceful transformation of the continent's security landscape. Yet ironically, the euphoria that came with the end of the Cold War has run its course. It has been replaced by cool calculation, shifting coalitions based on selfinterest, and the politics of the lowest common denominator. As the differences of opinion over the War in Iraq and the draft European constitution have made clear, Europe is by no means simply split into an »old « and a »new« camp. There is room for scepticism whether enlargement of EU and NATO will make it any easier to both to achieve internal consensus over goals and to resolve long-standing conflicts of interest between members.
\end{abstract}

Key Words: Enlargement EU/NATO, Euro-Atlantic Security, Eastern Europe

$\mathrm{W}$ e auch immer die Erweiterung der Nordatlantischen Allianz und der Europäischen Union einst rückblickend beurteilt wird, eines steht heute bereits fest: Das Jahr 2004 markiert eine Zäsur in der wechselvollen Geschichte Europas. Mit der weitreichenden Veränderung seiner sicherheitspolitischen Geographie wurde der durch den Zweiten Weltkrieg entstandene Riss zwischen Ost und West beseitigt. Ob die Erweiterung wirklich ein historischer Schritt zur Vereinigung Europas war, oder an anderer Stelle möglicherweise neue Bruchlinien von Dauer entstehen, bleibt künftiger

\footnotetext{
* Prof. Dr. Dr. Hans J. Giessmann ist stellvertretender Wiss. Direktor am Institut für Friedensforschung und Sicherheitspolitik an der Universität Hamburg.
}

Bewertung vorbehalten. Skepsis ist nicht unbegründet. Sie stützt sich auf die Beobachtung einer in den zurückliegenden fünf Jahren auffällig disproportionalen politischen und institutionellen Dynamik im euroatlantischen Binnenverhältnis. Von der Euphorie jener Gipfeltreffen, auf denen einst die Weichen für die Erweiterung gestellt wurden, ist hinter der zur Schau getragenen Fassade ungetrübter Feierstimmung nicht mehr viel zu spüren. Statt dessen dominieren nüchterne Interessenkalküle, wechselnde Koalitionen, Politikansätze des kleinsten gemeinsamen Nenners. Europa befindet sich zum wiederholten Male an einem Scheideweg. Die jüngsten Erfahrungen bieten Anlass zur Sorge, dass sich die verantwortlich Handelnden dessen nur ungenügend bewusst sind und 